\title{
A Pilot Study of Adjunctive Family Psychoeducation in Adolescent Major Depression: Feasibility and Treatment Effect
}

\author{
MARK SANFORD, M.B.Ch.B., MICHAEL BOYLE, Ph.D., LYNN MCCLEARY, Ph.D., \\ JENNIFER MILLER, B.Sc.N., MARGARET STEELE, M.D., ERIC DUKU, M.Sc., \\ AND DAVID OFFORD, M.D.†
}

\begin{abstract}
Objective: To obtain preliminary evidence of the feasibility and effectiveness of adjunctive family psychoeducation in adolescent major depressive disorder. Method: Participants were from outpatient clinics in Hamilton and London, Ontario. Over 24 months, 41 adolescents ages 13 through 18 years meeting major depressive disorder criteria were recruited (31 in Hamilton, 10 in London). Participants were randomized to usual treatment or usual treatment plus family psychoeducation. Outcome measures were readministered at 2 weeks, mid-treatment, posttreatment, and 3-month follow-up. Intent-to-treat analyses used $\chi^{2}$ and $t$ tests and growth curve analysis. Standardized effects based on growth curve estimates were calculated for continuous outcomes. Results: The London site was withdrawn because of poor participant retention. In Hamilton, no participant missed more than one assessment and there was good family psychoeducation adherence. Compared to controls, participants in the experimental group showed greater improvement in social functioning and adolescent-parent relationships (with medium standardized effect size $>0.5$ ), and parents reported greater satisfaction with treatment. Conclusions: There were positive treatment effects on family and social functioning processes postulated to mediate the clinical course of major depressive disorder. The study provides support for further evaluation of family psychoeducation in this clinical population. J. Am. Acad. Child Adolesc. Psychiatry, 2006;45(4):386-395. Key Words: major depression, family psychoeducation, adolescent, randomized, controlled trial.
\end{abstract}

Major depressive disorder (MDD) is a common disorder frequently beginning during adolescence,

Accepted November 8, 2005.

Dr. Sanford is with the Department of Psychiatry, University of Toronto, Toronto, Ontario; Dr. Boyle and Mr. Duku are with the Department of Psychiatry and Behavioural Neurosciences, McMaster University, Hamilton, Ontario; Dr. McCleary is with the Faculty of Nursing, Brock University, St Catherines, Ontario; Dr. Steele is with the Department of Psychiatry, University of Western Ontario, London; and Ms. Miller is with the Hamilton Health Sciences, Hamilton; Dr. Offord was with the Division of Child Psychiatry, McMaster University, Hamilton, Ontario, Canada.

$\dagger$ Deceased.

The study was supported by a grant from The Hospital for Sick Children Foundation, Toronto, Ontario.

Article Plus (online only) materials for this article appear on the Journal's Web site: www.jaacap.com.

Correspondence to Dr. Mark Sanford, Centre for Addiction and Mental Health, 250 College Street, Toronto, Ontario, Canada M5T 1R8; e-mail: Mark_Sanford@camh.net.

0890-8567/06/4504-0386@2006 by the American Academy of Child and Adolescent Psychiatry.

DOI: 10.1097/01.chi.0000198595.68820.10 and, like adults, referred depressed adolescents are at increased risk of mood and other psychiatric disorders, suicide, and impaired social functioning (Kovacs, 1996). Furthermore, it appears that the first depressive episode sets in motion changes in brain functioning, cognition, and social functioning that increase vulnerability to subsequent episodes (Harris, 2001; Post, 1992). It is likely that for many depressed adolescents, intensive multimodal treatments may be needed to alleviate acute symptoms, reverse neurochemical and cognitive concomitants of depression, and improve functioning within the social context.

Selective serotonin reuptake inhibitors (SSRIs; Emslie et al., 1998), cognitive-behavioral therapy (CBT; Brent et al., 1997; Wood et al., 1996), and interpersonal therapy (Mufson et al., 1999) are currently first-line treatments for referred depressed adolescents. The only large study to compare single with combined treatments showed that depressed adolescents had better 
outcomes with fluoxetine combined with CBT than with these treatments alone (Treatment for Adolescents with Depression Study, 2004). However, it is noteworthy that in most studies, up to $40 \%$ of referred cases do not respond to these treatments, and in some responders, residual symptoms persist over months and years (Brent et al., 2001; Emslie et al., 1998). Crosssectional studies consistently show high levels of parent-adolescent conflict, disturbance of affective bonds, and parent-adolescent alienation in families with a depressed adolescent (Patton et al., 2001). Family factors, including the presence of parental psychopathology and family dysfunction, are also associated with a worse clinical course of MDD, poor adherence to treatment, and poor treatment response (Birmaher et al., 1996; Brent et al., 1998). Thus, it is appropriate to consider whether including parents and other family members in treatment could substantially improve outcomes. Lewinsohn et al. (1990) compared CBT alone with CBT with a parent intervention component in referred adolescents. There were no statistically significant differences between groups, although the combined group did report fewer depressive symptoms at posttreatment. In another study of depressed and bipolar preadolescent children, six sessions of family psychoeducation (FPE) led to greater improvements in those receiving the program and improved adherence to other treatments (Fristad et al., 2002). Miklowitz et al. (2004) provided 21 sessions of family-focused psychoeducation as an adjunct to medications in an uncontrolled open trial involving 20 adolescents with bipolar disorder and reported improvements in depression, manic symptoms, and behavior problems over 1 year. In contrast, FPE was no more effective than routine follow-up in adolescents with MDD with intentional drug overdose but was associated with reduced suicidal ideation in cases without MDD (Harrington et al., 2000).

In summary, many depressed adolescents do poorly despite treatment and have serious disorders and impairments into adulthood. There is a paucity of research into whether there is added benefit to continuing treatments beyond initial acute treatment response (i.e., longer treatments versus brief time-limited "acutephase" interventions), or to combined treatments, and whether there are maintenance effects of treatments used alone or in combination. None of the proven treatments address directly the family context, although this appears to have a key influence on outcome. FPE is a proven effective adjunctive maintenance treatment in adults with psychiatric disorders (Miklowitz et al., 2000), is promising in depressed and bipolar preadolescent children and adolescents (Fristad et al., 2002; Miklowitz et al., 2004), but has not been evaluated in depressed adolescents. Therefore, this exploratory study into the feasibility and acute treatment effects of FPE in referred depressed adolescents is a timely first step in exploring the effectiveness of this approach.

The objectives of the study were (1) to assess the feasibility of adjunctive FPE in referred adolescents with MDD and (2) to estimate the acute treatment effects of FPE on depressive symptoms and family and social functioning outcomes (the postulated mediators of maintenance effects). It was assumed that medium standardized effect sizes (ESs; $d>0.5$ ) for the family process and social functioning outcomes would justify further maintenance treatment studies.

\section{METHOD}

\section{Participants}

The study was undertaken with review and monitoring by the Ethics Review Committees of Hamilton Health Sciences and of the London Health Sciences Centre. Participants were drawn from outpatient clinics serving depressed adolescents in Hamilton and London, Ontario, Canada. In Hamilton, participants were from a child and adolescent mood disorders clinic that was the main provider of outpatient services for this region (population 500,000). In London, referrals were sought from four inpatient and outpatient psychiatry programs serving adolescents in a geographic catchment area (population 350,000). All of the referrals were eligible after receiving a full description of the study and providing signed informed consent (with assent in participants younger than 16 years of age). Inclusion criteria were as follows: (1) meets DSM-IV MDD criteria (American Psychiatric Association, 1995) in the past 6 months according to both referring clinician and Schedule for Affective Disorders and Schizophrenia for School- Age ChildrenPresent Episode version (K-SADS-P); (2) lives with birth, adoptive, or stepparent; and (3) baseline assessment completed within 3 months of admission to clinic. Exclusion criteria were (1) history of mental retardation, pervasive developmental disorder, conduct disorder, or anorexia nervosa according to referring clinician and (2) nonaffective psychosis or mania by K-SADS-P interview.

\section{Instruments and Measures}

The K-SADS-P (Chambers et al., 1985) was used at baseline to determine study eligibility and comorbid internalizing disorders in the preceding 6 months. The depressive disorders and mania sections were used at posttreatment and follow-up to assess MDD remission/persistence and relapse/recurrence. The adolescent was the sole informant. The K-SADS-P sections provide DSM-III diagnoses, so modifications were made to elicit symptoms, symptom 
duration, and impairments required for DSM-IV diagnoses (e.g., assessment of impairment criteria for MDD episode).

The primary outcomes were measures of depressive symptoms, social functioning, family functioning, and adolescent relationship with parents. Depressive symptoms were measured using the Reynolds Adolescent Depression Scale (RADS; Reynolds, 1986). The 31 items are summed for a total score (range 31-124) and the recommended clinical cutoff is 78 . Adolescent social functioning was measured using the global score of the Structured Social Adjustment Interview (SSAI), a structured interview with good testretest reliability (McCleary and Sanford, 2002), adapted by the authors from the Social Adjustment Inventory for Children and Adolescents (John et al., 1987). Subscale scores (school, spare-time functioning, parent-adolescent and peer relationship domains) were used in secondary analyses. Family functioning was measured on the 12-item general functioning subscale of the Family Assessment Device (FAD; Epstein et al., 1983). The six other subscales of the FAD were used in secondary analyses. Adolescent relationships with mother and father were assessed using the Adjective Checklist (ACL; Friedmann and Goldstein, 1993). The ACL consists of 10 negative and 10 positive adjectives describing relationships, each rated by the adolescent on an 8-point Likert scale.

Secondary outcomes also included adolescent global functioning using the Children's Global Assessment Scale (CGAS; Shaffer et al., 1983), rated by the primary clinician, and adolescent and parent satisfaction with services using the Client Satisfaction Questionnaire (CSQ; Larsen et al., 1979).

Descriptive measures and data included sociodemographic data obtained using the Ontario Child Health Study Demographics Form (Boyle et al., 1986), self-reported drug use in the past 6 months using the Ontario Child Health Study Drug Use Survey (Boyle et al., 1986), and parent-rated disruptive behaviors using a DSM-IV Disruptive Behavior Disorders Checklist (an unpublished scale is available at the Journal's Web site at www.jaacap.com via the Article Plus feature).

\section{Monitoring Fidelity of FPE, Contamination, and Co-intervention}

The assessors gathered information from the adolescent and parent regarding hospitalizations, day treatment, medications (dose, duration of treatment, doses missed), and psychosocial interventions (type, number of sessions, sessions missed). Fidelity to FPE was assessed through clinician reports of number of sessions completed, family member attendance, and rated percentage of adherence to the curriculum components (education, communication, problem solving), noting reasons for failures in adherence.

\section{Design and Procedures}

Randomization and Follow-up Assessment Procedures. The study was an unblinded, randomized, controlled trial. Participants were randomly assigned to treatment conditions (usual treatment or usual treatment plus FPE) using a random numbers table to determine group assignment, with randomization in blocks of four to ensure a manageable flow to usual treatment plus FPE. Opaque sealed numbered envelopes containing assignment were opened in order after participants completed the baseline assessment. Study team members were blind to the blocking procedure. Randomization was by a statistician (M.B.) with no involvement in running the study.

The outcome measures were administered at baseline and readministered at 2 weeks (retest), at 3 months (mid-treatment),
6 months (posttreatment), and 9 months (follow-up) (for a total of five assessments during the course of the study). Beyond baseline, assessments were conducted in the home.

Family Psychoeducation. The family program used was developed by the authors and is described in a manual (available from the corresponding author). The intervention consists of twelve 90minute sessions conducted in the home, involving all family members living in the home who agree to participate, during the first 6 months after enrollment in the study, with one booster session at 3-month follow-up. The theoretical models (medical model, patient education, stress vulnerability, communication, and coping models) and the specific interventions used are consistent with those that have proven to be effective in families with a member with schizophrenia (Falloon et al., 1984), and more recently in patients with bipolar disorder and their families (Miklowitz et al., 2000). The approach is not intended for delivery by novice clinicians. The aims are to increase family knowledge about adolescent depression, to increase understanding of the experience of depression and its impact on the family, to strengthen family communication (to reduce hostile, critical, clinging, or isolating interactions and to foster supportive/warm interactions), and to enhance effective coping, problem solving, and management of crises and relapses.

There are five phases. The first session consists of an introduction to the therapy model, assessment of family factors relevant to the intervention, and goal setting. Sessions 2 through 4 focus on education about the clinical features, causes, treatment, and clinical course of adolescent depression. Sessions are structured, interactive, and involve direct communication between the adolescent and other family members about the experience of depression, resulting in greater empathy and understanding. Sessions 5 through 7 focus on communication skills training, identifying and minimizing unproductive interaction patterns and enhancing positive interactions. Sessions 8 through 11 focus on family problem-solving skills training, with weekly homework practice assigned. During session 12 , progress made over the course of therapy is reviewed and possible barriers to continued use of newly acquired skills are discussed. The final session is a booster, 2 to 3 months later, where progress in maintaining use of learned skills is assessed and strategies to sustain their use are identified.

Usual Treatment. All participants were offered usual treatments consisting of individual or group counseling and/or drug therapy with supportive case management. The first-line drug treatment was antidepressants (SSRIs, venlafaxine, or bupropion), if indicated in combination with anxiolytics or antipsychotics. These drugs were prescribed to adult therapeutic doses as tolerated, but there was no standardization of prescribing and no pill counts were conducted. All of the participants, regardless of whether they received medications, received handouts describing MDD, clinical presentation, course, treatment, and, in those receiving drugs, handouts about the prescribed medication. Usual psychosocial treatments were not standardized with respect to frequency of follow-up or fidelity to a specific treatment model.

\section{Analyses}

Primary Outcomes. The incremental effects on continuous outcome measures (RADS, FAD general functioning, ACL, SSAI global social functioning scores) attributable to treatment grouping were assessed using growth curve analysis based on hierarchical linear modeling (HLM; Bryk and Raudenbush, 1992). There were two steps. First, within-subject changes on outcome measures were modeled as a function of time. Straight-line growth models 
(modeled as a linear function of the square root of time) were fit separately to each measure observed for each subject at each assessment (using data from all five assessments). Second, the withinsubject parameter estimates or slopes defining the trajectories of change in outcome for each individual were used as the dependent variables for evaluating differences between treatment groups. A standardized ES of 0.5 between treatment groups was selected as the minimally important ES. The growth curve analyses were used to determine whether this difference was realized for each outcome. The ES was calculated for each of the outcomes based on modeled estimates of scores at baseline, posttreatment, and follow-up using the following formula: $\mathrm{ES}$ = change in experimental group from baseline - change in control group from baseline/baseline SD.

Secondary Outcomes (Exploratory Analyses). Chi-square tests were used to compare MDD remission rates between experimental and control groups at posttreatment and follow-up. A $t$ test was used to compare experimental and control groups on CSQ scores. Finally, growth curve analyses were conducted on CGAS, FAD subscales, and SSAI subscales to test assumptions about expected group differences.

\section{RESULTS}

\section{Sampling, Randomization, Adherence to Treatment}

In London, there were 10 referrals, but because of poor adherence, baseline data were complete for four subjects and only two were followed beyond the midtreatment assessment. Therefore, this site has been dropped from the study and the description that follows refers to the Hamilton site alone. This decision was made because none of the London participants received more than one FPE session; in this small study, including data from these subjects would have rendered the findings impossible to interpret. In Hamilton, over a 24-month period (January 1999-December 2000), there were 58 eligible referrals and 31 participants (53.4\%). Of the 27 nonparticipants, 10 refused family involvement (6 adolescents and 4 parents), 1 refused in-home delivery, 2 refused randomization, 6 were satisfied with services and wanted no more treatment, and 8 were missed or dropped out of clinical care before they could be recruited to the study. Following randomization, there were 16 and 15 participants in experimental and control groups, respectively. Posttreatment assessments were completed on 14 experimental participants and 14 controls ( 3 missed), and all 31 participants were assessed at 3-month follow-up. The Hamilton data used in the analyses are close to complete over the full extent of the study.

Table 1 provides the demographic and baseline clinical characteristics for the 31 participants. Approximately two thirds were girls, and participants were most commonly in the mid-adolescent age range (mean age
15.9 years). The families showed little indication of social disadvantage (61.3\% were two-parent families, $9.7 \%$ reported total family income $<\$ 25,000$, and $87.1 \%$ of primary parent informants had completed secondary education). More than two thirds of the adolescents reported a clear precipitant before the onset of MDD. Many were depressed over an extended period (mean duration of MDD 58.9 weeks), and approximately one third reported a previous depressive episode. There was a high rate of comorbid disorders, especially generalized anxiety disorder and social phobia

\section{TABLE 1}

ample Characteristics

\begin{tabular}{|c|c|c|c|}
\hline Private Characteristic & $\begin{array}{c}\text { Treatment } \\
(n=16)\end{array}$ & $\begin{array}{l}\text { Control } \\
(n=15) \\
\end{array}$ & $\begin{array}{c}\text { Total } \\
(n=31)\end{array}$ \\
\hline Female (\%) & $9(56.2)$ & $11(73.3)$ & $20(64.5)$ \\
\hline Mean age, yr (SD) & $15.6(1.1)$ & $16.1(1.6)$ & $15.9(1.4)$ \\
\hline $\begin{array}{l}\text { Single-parent } \\
\text { family }(\%)\end{array}$ & $7(43.7)$ & $5(33.3)$ & $12(38.7)$ \\
\hline Income $<\$ 25,000(\%)$ & $2(12.5)$ & $1(6.7)$ & $3(9.7)$ \\
\hline Parent $\geq$ grade $12(\%)$ & $14(87.5)$ & $13(86.7)$ & $27(87.1)$ \\
\hline $\begin{array}{l}\text { Partner } \geq \text { grade } 12 \\
\quad(n=25)(\%)\end{array}$ & $9(81.8)$ & $9(64.3)$ & $18(72.0)$ \\
\hline MDE precipitant (\%) & $10(62.5)$ & $10(66.7)$ & $20(64.5)$ \\
\hline $\begin{array}{l}\text { Mean duration of } \\
\text { MDE (SD) }\end{array}$ & $58.9(37.0)$ & $59.0(88.6)$ & $58.9(66.7)$ \\
\hline Past MDE (\%) & $5(31.2)$ & $7(46.7)$ & $12(38.7)$ \\
\hline $\begin{array}{l}\text { MDD with } \\
\text { psychosis (\%) }\end{array}$ & $2(12.5)$ & $2(13.3)$ & $4(12.9)$ \\
\hline $\begin{array}{l}\text { Dysthymic } \\
\text { disorder (\%) }\end{array}$ & $3(18.7)$ & $3(20.0)$ & $6(19.3)$ \\
\hline $\begin{array}{l}\text { Separation anxiety } \\
\text { disorder }(\%)\end{array}$ & $1(6.2)$ & $3(20.0)$ & $4(12.9)$ \\
\hline Social phobia (\%) & $11(68.7)$ & $8(53.3)$ & $19(61.3)$ \\
\hline $\begin{array}{l}\text { Generalized anxiety } \\
\text { disorder (\%) }\end{array}$ & $6(37.5)$ & $8(53.3)$ & $14(45.1)$ \\
\hline OCD (\%) & $6(37.5)$ & $1(7.7)^{a}$ & $7(22.6)$ \\
\hline $\begin{array}{l}\text { Posttraumatic stress } \\
\text { disorder }(\%)\end{array}$ & $2(12.5)$ & $3(20.0)$ & $5(16.1)$ \\
\hline $\begin{array}{l}\text { Drunk in past } \\
6 \mathrm{mo}(\%)\end{array}$ & $7(43.7)$ & $10(66.7)$ & $17(54.8)$ \\
\hline $\begin{array}{l}\text { Cigarettes } \geq 10 \\
\text { daily }(\%)\end{array}$ & $4(25.0)$ & $3(20.0)$ & $7(22.6)$ \\
\hline Cannabis use (\%) & $10(62.5)$ & $9(60.0)$ & $19(61.3)$ \\
\hline Hallucinogen use (\%) & $4(25.0)$ & $1(7.7)$ & $5(16.1)$ \\
\hline $\begin{array}{r}\text { Mean CGAS } \\
\text { score (SD) }\end{array}$ & $48.9(10.4)$ & $54.7(7.7)$ & $51.7(9.5)$ \\
\hline
\end{tabular}

Note: $\mathrm{MDE}=$ major depressive episode; $\mathrm{MDD}=$ major depressive disorder; CGAS = Children's Global Assessment Scale.

${ }^{a}$ Significant difference between experimental and control groups $(p<.05)$. 
(45.1\% and $61.3 \%$, respectively). There was also a high rate of substance use in the preceding 6 months; more than half of the participants reported having been drunk, $22.6 \%$ smoked $\geq 10$ cigarettes daily, and many reported use of illicit drugs, both cannabis and hallucinogens $(61.3 \%$ and $16.1 \%$, respectively). There were no significant differences between the groups for parentrated disruptive behaviors on the Disruptive Behaviours Checklist (not shown in the table). A high level of global disturbance was reflected in the baseline CGAS scores (mean score $=51.7, \mathrm{SD}=9.5$, with 60 suggested as the usual clinical cutoff). Testing for differences between the two treatment groups indicates that they were similar postrandomization with only one significant difference, a higher rate of obsessive-compulsive disorder in the experimental group $\left(\chi^{2}=4.2, d f=3, p<.05\right)$, and lower but not significantly different CGAS scores in the experimental group $(t=1.75, d f=29, p<.1)$.

In the experimental group, FPE sessions completed were as follows: 3 sessions (2 families), 5 sessions (1 family), 9 sessions ( 2 families), 10 sessions (2 families), 11 sessions ( 1 family), 12 sessions ( 8 families). Therefore, 13 of 16 families $(81 \%)$ completed $\geq 9$ of the possible 12 FPE sessions. Based on the clinicians' evaluation of adherence to the program, the mean percentage of completion for each of the FPE components were (1) education, 83.9 ( $S D=20.1)$; (2) communication skills, 66.4 ( $\mathrm{SD}=31.0$ ); (3) problem-solving skills, 61.0 ( $S D=27.8)$. At baseline, 26 of the participants were receiving antidepressants (13 experimental, 13 controls) and 27 were receiving individual counseling (13 experimental, 14 controls; 6 CBT, 20 supportive therapy, 1 interpersonal therapy IPT). At posttreatment, 20 participants were continuing with antidepressants (13 experimental, 7 controls $\chi^{2}=2.5, d f=3, p=.1$ ). Twenty-two participants ( 11 experimental, 11 controls) had received individual therapy in the 3 months since the midtreatment assessment. By follow-up, 22 participants (12 experimental, 10 controls) were either continuing treatment with antidepressants or had restarted them $\left(\chi^{2}=0.3, d f=3, p=\right.$ not significant $)$. Twenty-four participants (12 experimental, 12 controls) reported having individual counseling sessions in the 3 months since the posttreatment assessment.

\section{Primary Outcomes}

Table 2 provides parameter estimates from growth curves for each of the primary outcomes with the statistical significance of between-group differences indicated in the far right column. The trajectory columns indicate the actual unit change in the dependent variable per unit of time (in this instance, the time unit was 3 months, the period between assessments). For example, row 1 shows that the RADS score (adolescent informant) decreased by 2.6 and 5.7 during each 3-month period in controls and experimental groups, respectively. This difference in trajectory between groups approached statistical significance $(p=.052)$. In five of the social and family functioning outcomes, group trajectories were significantly different (negative adjectives score for adolescent-mother relationship, both positive and negative adjective scores for adolescent-father relationship, and adolescent global social functioning scores according to both adolescent and parent informants). In each instance, the

TABLE 2

Parameter Estimates for Primary Outcomes

\begin{tabular}{|c|c|c|c|c|}
\hline \multirow{2}{*}{$\begin{array}{c}\text { Private } \\
\text { Outcomes }\end{array}$} & \multicolumn{2}{|c|}{ Private Baseline } & \multicolumn{2}{|c|}{ Trajectory } \\
\hline & Control & Experimental & Control & Experimental \\
\hline RADS TS & 78.2 & 79.9 & -2.6 & $-5.7(p=.052)$ \\
\hline $\begin{array}{l}\text { FAD GF } \\
\text { adolescent }\end{array}$ & 2.56 & 2.37 & -0.06 & $-0.12^{2}$ \\
\hline $\begin{array}{r}\text { FAD GF } \\
\text { parent }\end{array}$ & 2.26 & 2.18 & -0.04 & -0.01 \\
\hline $\begin{array}{l}\text { SSAI GF } \\
\text { adolescent }\end{array}$ & 98.56 & $88.75^{*}$ & 1.26 & $6.11^{* *}$ \\
\hline $\begin{array}{l}\text { SSAI GF } \\
\text { parent }\end{array}$ & 97.27 & $82.53^{*}$ & 1.49 & $7.09^{* *}$ \\
\hline $\begin{array}{l}\mathrm{ACL} \\
\text { mother pos }\end{array}$ & 57.47 & 55.71 & 0.15 & 1.33 \\
\hline $\begin{array}{l}\text { ACL } \\
\text { mother neg }\end{array}$ & 38.2 & 36.7 & -0.14 & $-2.40^{*}$ \\
\hline $\begin{array}{l}\text { ACL } \\
\text { father pos }\end{array}$ & 54.48 & 47.5 & -0.67 & $5.19^{* *}$ \\
\hline $\begin{array}{l}\text { ACL } \\
\text { father neg }\end{array}$ & 41.6 & 45.8 & 0.17 & $-5.16^{* *}$ \\
\hline
\end{tabular}

Note: $p$ Values are for comparisons between experimental and control (i.e., how different the differences in parameter estimates are from zero). RADS TS = Reynolds Adolescent Depression Scale total score; FAD GF = Family Assessment Device General Functioning Subscale score; SSAI GF = Structured Social Adjustment Interview Global Functioning score; ACL mother pos = Adjective Checklist mother positive score; ACL mother neg = Adjective Checklist mother negative score; ACL father pos = Adjective Checklist father positive score; ACL father neg = Adjective Checklist father negative score.

$$
{ }^{*} p<.05 ;{ }^{* *} p<.001 \text {. }
$$


experimental group moved further than controls toward more positive functioning and relationships. As indicated in column 3, there were significant betweengroup differences at baseline in global social functioning scores (for both adolescent and parent reports), with the experimental group functioning worse than controls.

Table 3 provides the estimated mean scores along with the ES for the outcome variables. The ES of $d<0.5$ were found for six of nine outcomes both at posttreatment and at 3-month follow-up. The largest effects $(d>0.9)$ were seen in measures of global social functioning (both informants) and in the adolescent's perception of relationship with father on the ACL. Considering the ES for all outcomes, there was a positive effect favoring the experimental group for eight of nine outcomes both at posttreatment and follow-up (the FAD general functioning score by parent informant being the only exception). Graphic representations of the growth curve analyses (with clinical cut points indicated with dotted lines) are available on the Journal's Web site at www.jaacap.com via the Article Plus feature.

\section{Secondary Outcomes}

At baseline, MDD criteria were met by $75 \%$ and $67 \%$ of experimental participants and controls, respectively. The remaining participants met criteria for admission to the study (MDD within 6 months of baseline) but had reached partial or full remission at baseline. At posttreatment, 21\% of experimental participants and $50 \%$ of controls met MDD criteria (unremitted cases), whereas at follow-up, 25\% of experimental participants and $53 \%$ of controls met criteria. The differences at follow-up favor the experimental group with a trend toward significance $\left(\chi^{2}=2.6\right.$, $d f=3, p=.1)$.

Post hoc, growth curve analyses were performed for global functioning (CGAS) scores and subscale scores of the SSAI (school performance, school problems, spare-time activities, adolescent-mother relationship, adolescent-father relationship, and peer relationships) and the FAD (problem solving, communication, roles, affective involvement, affective responsiveness, and behavior control). The results of these analyses are summarized in Table 4. The scales that showed significant differences in trajectory were as follows: for adolescent informant, the SSAI adolescent-mother relationship, adolescent-father relationship, and peer relationships subscales and the FAD communication and affective involvement subscales; for the parent informant, the SSAI adolescent-father relationship and the FAD roles subscale with improvement favoring controls. In all but one instance, the experimental participants made more positive change than controls. The trajectory for improvement on the CGAS was not significantly different between treatment groups.

Finally, mean CSQ scores were compared to assess participant satisfaction with services. The experimental group parents were more satisfied than controls,

TABLE 3

Estimated Mean Outcome Scores at Baseline and at 3, 6, and 9 Months and Standardized Effect Sizes at 6 and 9 Months

\begin{tabular}{|c|c|c|c|c|c|c|c|c|c|c|c|}
\hline \multirow[b]{2}{*}{ Outcomes } & \multirow[b]{2}{*}{ Baseline SD } & \multicolumn{4}{|c|}{ Control } & \multicolumn{4}{|c|}{ Experimental } & \multicolumn{2}{|c|}{ Effect Size } \\
\hline & & Baseline & $3 \mathrm{Mo}$ & $6 \mathrm{Mo}$ & $9 \mathrm{Mo}$ & Baseline & $3 \mathrm{Mo}$ & $6 \mathrm{Mo}$ & $9 \mathrm{Mo}$ & $6 \mathrm{Mo}$ & $9 \mathrm{Mo}$ \\
\hline RADS TS & 14.4 & 78.2 & 73.6 & 71.7 & 70.3 & 79.9 & 70.0 & 65.9 & 62.8 & 0.52 & 0.64 \\
\hline FAD GF adolescent & 0.7 & 2.6 & 2.5 & 2.4 & 2.4 & 2.4 & 2.2 & 2.1 & 2.1 & 0.1 & 0.25 \\
\hline FAD GF parent & 0.4 & 2.3 & 2.2 & 2.2 & 2.1 & 2.2 & 2.2 & 2.1 & 2.1 & -0.19 & -0.19 \\
\hline SSAI GF adolescent & 12.7 & 98.6 & 100.7 & 101.6 & 102.3 & 88.7 & 99.3 & 103.7 & 107.1 & 0.93 & 1.14 \\
\hline SSAI GF parent & 14.3 & 97.3 & 99.8 & 100.9 & 101.7 & 82.5 & 94.8 & 99.9 & 103.8 & 0.96 & 1.17 \\
\hline ACL mother pos & 16.3 & 57.5 & 57.7 & 57.8 & 57.9 & 55.7 & 58 & 59 & 59.7 & 0.17 & 0.22 \\
\hline ACL mother neg & 10.7 & 38.2 & 38 & 37.9 & 37.8 & 36.7 & 32.6 & 30.8 & 29.5 & 0.52 & 0.63 \\
\hline ACL father pos & 19.6 & 54.5 & 53.3 & 52.8 & 52.5 & 47.5 & 56.5 & 60.2 & 63.1 & 0.73 & 0.90 \\
\hline ACL father neg & 13.9 & 41.6 & 41.9 & 42 & 42.1 & 45.8 & 37 & 33.4 & 30.6 & 0.92 & 1.1 \\
\hline
\end{tabular}

Note: Net effect size = change in treatment (from baseline) - change in control (from baseline)/baseline SD. RADS TS = Reynolds Adolescent Depression Scale total score; FAD GF = Family Assessment Device General Functioning Subscale score; SSAI GF = Structured Social Adjustment Interview Global Functioning score; ACL mother pos = Adjective Checklist mother positive score; ACL mother neg = Adjective Checklist mother negative score; ACL father pos = Adjective Checklist father positive score; ACL father neg = Adjective Checklist father negative score. 
TABLE 4

Parameter Estimates for Secondary Outcomes

\begin{tabular}{|c|c|c|c|c|}
\hline & \multicolumn{2}{|c|}{ Baseline } & \multicolumn{2}{|c|}{ Trajectory } \\
\hline & Control & Experimental & Control & Experimental \\
\hline \multicolumn{5}{|l|}{ SSAI adolescent } \\
\hline School performance & 13.45 & 12.36 & -0.30 & -0.04 \\
\hline School problems & 11.12 & 9.63 & 0.70 & 2.13 \\
\hline Spare-time activities & 14.78 & 12.54 & -0.16 & 0.47 \\
\hline Adolescent-mother relationship & 24.93 & 24.41 & 0.21 & $1.16^{a}$ \\
\hline Adolescent-father relationship & 24.51 & 24.07 & 0.09 & 1.25 \\
\hline Peer relationship & 28.47 & $24.20^{a}$ & -0.22 & -0.80 \\
\hline \multicolumn{5}{|l|}{ SSAI parent } \\
\hline School performance & 12.90 & 11.82 & -0.16 & 0.10 \\
\hline School problems & 10.85 & 7.92 & 0.80 & 2.22 \\
\hline Spare time activities & 12.75 & 11.48 & -0.04 & 0.55 \\
\hline Adolescent-mother relationship & 27.54 & 24.54 & 0.25 & 1.17 \\
\hline Adolescent-father relationship & 25.24 & 21.05 & 0.21 & $1.77^{a}$ \\
\hline Peer relationship & 26.65 & $22.56^{a}$ & 0.42 & 0.98 \\
\hline \multicolumn{5}{|l|}{ FAD adolescent } \\
\hline Problem solving & 2.65 & 2.51 & -0.02 & -0.10 \\
\hline Roles & 3.08 & 3.13 & -0.07 & -0.34 \\
\hline Communication & 2.52 & 2.53 & 0.01 & $-0.12^{a}$ \\
\hline Affective involvement & 2.48 & 2.42 & -0.00 & $-0.10^{a}$ \\
\hline Affective responsiveness & 2.47 & 2.48 & -0.00 & -0.11 \\
\hline Behavior control & 2.08 & 2.16 & -0.03 & -0.11 \\
\hline \multicolumn{5}{|l|}{ FAD parent } \\
\hline Problem solving & 2.31 & 2.25 & -0.04 & -0.00 \\
\hline Roles & 3.37 & $2.51^{a}$ & -0.35 & $-0.04^{a}$ \\
\hline Communication & 2.28 & 2.27 & -0.03 & 0.00 \\
\hline Affective involvement & 2.30 & 2.25 & -0.03 & 0.02 \\
\hline Affective responsiveness & 1.97 & 2.22 & 0.02 & -0.06 \\
\hline Behavior control & 1.83 & 1.82 & -0.00 & -0.04 \\
\hline \multicolumn{5}{|l|}{ Children's Global Assessment Scale } \\
\hline Global score & 54.51 & 49.31 & 1.49 & 3.26 \\
\hline
\end{tabular}

Note: Recommended clinical cut points for FAD subscales: Problem Solving $=2.2$, Roles $=2.3$, Communication $=2.2$, Affective Involvement $=2.1$, Affective Responsiveness $=2.1$, Behavior Control = 1.9. SSAI $=$ Structured Social Adjustment Interview; FAD = Family Assessment Device.

${ }^{a}$ Significant difference between groups $(p<.05)$ at baseline (column 2) or in trajectory (column 4$)$.

whereas there was no difference between groups for the adolescent informant. The parent's mean CSQ scores were $29.4(\mathrm{SD}=4.8)$ and $24.5(\mathrm{SD}=4.9)$ in experimental and control groups, respectively $(t=$ 2.7, $d f=29, p<0.01)$. For the adolescent informant, the mean scores were $26.3(\mathrm{SD}=3.6)$ and $25.1(\mathrm{SD}=$ $3.0)$ in experimental and control groups, respectively $(t=1.0, d f=29, p=\mathrm{NS})$.

\section{DISCUSSION}

The first objective was to explore the feasibility of FPE in adolescent MDD. In Hamilton, depressed adolescents were recruited from recent clinic referrals and more than half of eligible referrals agreed to participate. Once involved in the study, no families dropped out and FPE participation rates were high. The participants had moderate to severe depression, as evidenced by low CGAS scores, high rates of comorbid disorders, and a long duration of the index MDD episode. In FPE families, the parents also reported greater satisfaction with treatment services than controls, indicating that families were positive about being actively involved in the treatment. In London, however, there were problems in recruiting and retaining families. These problems were not 
chiefly a reflection of low interest in family interventions or poor adherence to FPE, but demonstrate the difficulties of conducting clinical research in this population. There were many organizational and service differences between sites that could account for these discrepancies. The Hamilton site was home to the team that initiated the study and who were more experienced in using FPE, clinical psychosocial research was more established there, and a single clinical service provided all of the participants, whereas in London, referrals were sought from several programs and from a larger geographic catchment area. Psychoeducational approaches for emotionalbehavioral disorders were an established component of clinical services in Hamilton and were well accepted by frontline clinical staff, whereas in London, psychodynamic and family systems models were the predominant psychosocial treatment approaches. For whatever reason, the failure of the study in London shows that even if FPE is proven to be effective, transferring it may prove difficult, as it is for adult disorders (MacFarlane et al., 2001).

The second objective was to explore the efficacy of adjunctive FPE in depressed adolescents. Although this was a small study, there were significant differences between the experimental group and controls on the primary outcomes, consistently favoring the FPE group. First, the growth curve analyses demonstrated strong treatment effects in social functioning and family relationship domains. Parents reported improved adolescent social functioning, and post hoc analyses showed that this was accounted for largely by improvements in adolescent-parent relationships, suggesting a degree of specificity to the detected FPE effects. The adolescent's perception of the affective tone in their relationship with their parents was positively affected by FPE, especially perceptions of relationships with the father. However, there were no significant differences between groups on the general functioning subscale of the FAD. It is unclear whether this results from a lack of sensitivity to change of this subscale or whether general functioning is less affected by the intervention than specific areas of family functioning, such as communication and affective involvement, which were significantly improved in the experimental group according to the adolescent informant. There were trends to significance for decline of depressive symptoms and MDD persistence/remission. In these domains, the effects were medium (e.g., standardized ES at follow-up $=0.64$ for depressive symptom score). Overall, the findings are promising because they suggest that FPE influences processes that are postulated to affect the clinical course of adolescent depression. The chief rationale for studying FPE is that it may prove effective in preventing MDD recurrence, and to achieve this end, it will need to affect depressogenic processes. FPE attempts to strengthen the adolescent's social functioning, family relationships, family support, and coping. Although there is overlap in objectives and techniques with other family therapies, there are differences in the approach to the family, so it is not clear whether findings can be generalized to other family therapy approaches. Further research is required to test FPE against other family interventions to tease out which components of the intervention mediate change and to test maintenance effects. It is important to note that the positive findings reported here are not consistent with those of an earlier negative study of adolescents referred with overdose (Harrington et al., 2000). It is unclear whether sampling differences, the fact that in this study FPE was adjunctive to other treatments, or differences in the FPE method account for this discrepancy. In this study, referrals with conduct disorder were excluded because alternative parenttraining approaches are indicated in these cases. As a result, the findings reported here are not generalizable to depressed adolescents with comorbid conduct disorder who make up a less treatment-responsive population than those with comorbid anxiety disorder (Jayson et al., 1998).

\section{Limitations}

There are several limitations to this small exploratory trial. First, the small sample size results in lower accuracy in estimating treatment effects. The fact that there were statistically significant differences speaks to the medium to strong effects observed. Because of small numbers and the exploratory nature of the study, there was no statistical control of testing of multiple outcomes. Although this is appropriate at this stage of evaluation of a new intervention, type I errors may have occurred. Second, participants were not blinded to FPE or control conditions, the CGAS was rated by the primary clinician rather than an independent evaluator, and fidelity to the FPE intervention was self-rated by the therapists rather than through independent 
observation of tapes, transcripts, or observed sessions. These procedures could lead to reporting and attention biases. For instance, the therapists may have overestimated fidelity to the intervention, and participants in the experimental group may have provided biased responses on the questionnaires measuring primary outcomes, although one would expect attenuation of these effects during follow-up and this was not observed in the data. The K-SADS-P semistructured interview used to assess psychiatric disorder including the depressive disorder outcomes was based entirely on interview of the adolescent informant. It is likely that MDD rates would be different if parent interviews had been used as well (e.g., adolescents may have under- or overreported depression at posttreatment and followup) and parent-adolescent discrepancies may have arisen. However, there is no reason to expect that reporting discrepancies would be different between treatment and control participants. Third, there were baseline differences between treatment groups in global social functioning and overall functioning despite randomization. The experimental group was functioning worse than controls, so it remains possible that greater improvements in the FPE group reflect greater attenuation of disturbance over time. That is, that the experimental group simply improved back to mean symptom and impairment levels during followup, independent of any treatment effect. It is unlikely this could be a sole explanation of differences as in virtually all instances, experimental participants improved beyond scores achieved by controls, and baseline differences were controlled for in the growth curve analyses. Finally, the psychosocial and drug treatments were not standardized and we cannot report on dose received and adherence, although psychotropic medications were initiated and tolerated at usual adult doses. Those receiving FPE were more likely than controls to continue antidepressant medication throughout the study, although this difference was not statistically significant. However, improved adherence to medications in the FPE group may have contributed to better outcomes. This appeared to be the case in an earlier study of FPE in preadolescent children with bipolar disorder or major depression that found that those receiving FPE increased their use of clinical services, adhered better to treatment, and had better outcomes than controls (Fristad et al., 2002).

\section{Clinical Implications}

The clinical significance of the detected effects is uncertain. Although it is unknown whether the detected changes in symptoms and family relationships will translate into a more positive longer-term clinical course, an acute impact on social and family functioning is apparent. Studies to date suggest that combined treatment approaches are necessary in more complex cases and FPE could be one treatment component. However, FPE is a relatively intensive intervention and costly in terms of time involvement of the family and in clinical resources. In this study, FPE was delivered in the home, whereas others have used clinic-based approaches more readily transferrable to standard clinical services. The investigators opted for an in-home model predominantly for methodological reasons (to preserve power in a small study by reducing dropout rates, maintaining good treatment adherence, and reducing lost data). It is uncertain whether similar effects would be obtained with FPE delivered in the clinic.

In summary, this trial in depressed adolescents provides preliminary evidence that FPE improves adolescent social functioning, especially in the domain of adolescent-parent relationships, and may bring about a more rapid resolution of the MDD episode. In a clinical research setting with a committed study team, it is well accepted and there is good adherence to the intervention, but questions remain about its transferability to other clinical settings. The study does provide strong support for continuing investigation of FPE in this clinical population; especially needed are well-controlled studies into acute and maintenance treatment effects and identification of treatment modifiers. Once described effects are confirmed by other investigators, subsequent studies should focus on identifying treatment mediators, disentangling effects of the various intervention components, including attention effects, and determining minimum doses required to obtain a clinically important effect.

Disclosure: Dr. Sanford received a stipend for presenting on bipolar disorder at a drug company-sponsored continuing education event in 2004 (Abbott). Dr. Steele receives research funding, participates in national advisory board meetings, receives unrestricted educational grants, or serves on speakers' bureaus for Janssen, Eli Lilly, and Shire; she is also a consultant for CMED, a division of Omnicon Canada Inc. The other authors have no financial relationships to disclose. 


\section{REFERENCES}

American Psychiatric Association (1995), Diagnostic and Statistical Manual of Mental Disorders, 4th edition (DSM-IV). Washington, DC: American Psychiatric Association

Birmaher B, Ryan ND, Williamson DE, Brent DA, Kaufman J (1996), Childhood and adolescent depression: a review of the past 10 years: II. J Am Acad Child Adolesc Psychiatry 35:1575-1583

Boyle MH, Offord DR, Hofmann HG et al. (1986), Ontario Child Health Study: methodology. Arch Gen Psychiatry 44:826-831

Brent DA, Birmaher B, Kolko D, Baugher M, Bridge J (2001), Subsyndromal depression in adolescents after a brief psychotherapy trial: course and outcome. J Affect Disord 63:51-58

Brent DA, Holder D, Kolko D et al. (1997), A clinical psychotherapy trial for adolescent depression comparing cognitive, family, and supportive therapy. Arch Gen Psychiatry 54:877-885

Brent DA, Kolko DJ, Birmaher B, Baugher M, Bridge J, Roth C, Holder D (1998), Predictors of treatment efficacy in a clinical trial of three psychosocial treatments for adolescent depression. J Am Acad Child Adolesc Psychiatry 37:906-914

Bryck AS, Raudenbush SW (1992), Hierarchical Linear Models. Newbury Park, CA: Sage Publications

Chambers W, Puig-Antich J, Hirsch M et al. (1985), The assessment of affective disorders in children and adolescents by semi-structured interview: test-retest reliability of the Schedule for Affective Disorders and Schizophrenia for School-Age Children, Present Episode version. Arch Gen Psychiatry 42:696-702

Emslie GJ, Rush AJ, Weinberg WA, Kowatch RA, Carmody T, Mayes TL (1998), Fluoxetine in child and adolescent depression: acute and maintenance treatment. Depress Anxiety 7:32-39

Epstein NB, Baldwin LM, Bishop DS (1983), The McMaster Family Assessment Device. J Marital Fam Ther 9:171-180

Falloon IRH, Boyd JL, McGill CW (1984), Family Care of Schizophrenia. New York: The Guilford Press

Friedmann MS, Goldstein MJ (1993), Relatives awareness of their own expressed emotion as measured by a self-report adjective checklist. Fam Process 32:459-471

Fristad MA, Goldberg-Arnold JS, Gavazzi SM (2002), Multi-family psychoeducation groups (MFPG) for families of children with bipolar disorder. Bipolar Disord 4:254-262

Harrington R, Kerfoot M, Dyer E et al. (2000), Deliberate self-poisoning in adolescence: why does a brief family intervention work in some cases and not others? J Adolesc 23:13-20

Harris T (2001), Recent developments in understanding the psychosocial aspects of depression. Br Med Bull 57:17-32

Jayson D, Wood A, Kroll L, Fraser J, Harrington R (1998), Which depressed patients respond to cognitive-behavioral treatment? J Am Acad Child Adolesc Psychiatry 37:35-39

John K, Gammon GD, Prusoff BA, Warner V (1987), The Social Adjustment Inventory for Children and Adolescents (SAICA): testing of a new semi-structured interview. J Am Acad Child Adolesc Psychiatry 26:898-911

Kovacs M (1996), Presentation and course of major depressive disorder during childhood and later years of the life span. J Am Acad Child Adolesc Psychiatry 35:705-715

Larsen DL, Atkisson CC, Hargreaves WA, Nguyen TD (1979), Assessment of client/patient satisfaction: development of a general scale. Eval Program Plann 2:197-207

Lewinsohn PM, Clarke GN, Hops H, Andrews J (1990), Cognitivebehavioral treatment for depressed adolescents. Behav Ther 21:385-401

MacFarlane WR, McNacy S, Dixon L, Hornby H, Cimett E (2001), Prediction of dissemination of family psychoeducation in community health centres in Maine and Illinois. Psychiatr Serv 52:935-942

McCleary L, Sanford M (2002), Parental expressed emotion in depressed adolescents: prediction of clinical course and relationship to comorbid disorders and social functioning. J Child Psychol Psychiatry 43:587- 595

Miklowitz DJ, George EL, Axelson DA et al. (2004), Family-focused treatment for adolescents with bipolar disorder. J Affect Disord 82(suppl 1):S113-S128

Miklowitz DJ, Simoneau TL, George EL et al. (2000), Family-focused treatment for bipolar disorder: 1-year effects of a psychoeducational program in conjunction with pharmacotherapy. Biol Psychiatry 48:582-592

Mufson L, Weissman MM, Moreau D, Garfinkel R (1999), Efficacy of interpersonal psychotherapy for depressed adolescents. Arch Gen Psychiatry 56:573-579

Patton GG, Coffey C, Posterino M, Carlin JB, Wolfe R (2001), Parental "affectionless control" in adolescent depressive disorders. Soc Psychiatry Psychiatr Epidemiol 36:475-488

Post RM (1992), Transduction of stress into the neurobiology of recurrent affective disorder. Am J Psychiatry 149:999-1010

Reynolds W (1986), Assessment of Depression in Adolescents: Manual for the Reynold's Adolescent Depression Scale. Odessa, FL: Psychological Assessment Resources

Shaffer D, Gould MS, Brasic J et al. (1983), A Children's Global Assessment Scale (CGAS). Arch Gen Psychiatry 40:1228-1231

Treatment for Adolescents With Depression Study (TADS) Team (2004), Fluoxetine, cognitive-behavioral therapy, and their combination for adolescents with depression: Treatment for Adolescents with Depression Study (TADS) randomized controlled trial. JAMA 292:807-820

Wood A, Harrington R, Moore A (1996), A controlled trial of a brief cognitive-behavioral intervention in adolescent patients with depressive disorders. J Child Psychol Psychiatry 37:737-746

Psychosocial Implications of Disaster or Terrorism on Children: A Guide for the Pediatrician J.F. Hagan, Jr., and the Committee on Psychosocial Aspects of Child and Family Health and the Task Force on Terrorism

During and after disasters, pediatricians can assist parents and community leaders not only by accommodating the unique needs of children but also by being cognizant of the psychological responses of children to reduce the possibility of longterm psychological morbidity. The effects of disaster on children are mediated by many factors including personal experience, parental reaction, developmental competency, gender, and the stage of disaster response. Pediatricians can be effective advocates for the child and family and at the community level and can affect national policy in support of families. In this report, specific children's responses are delineated, risk factors for adverse reactions are discussed, and advice is given for pediatricians to ameliorate the effects of disaster on children. Pediatrics 2005;116:787-795. 